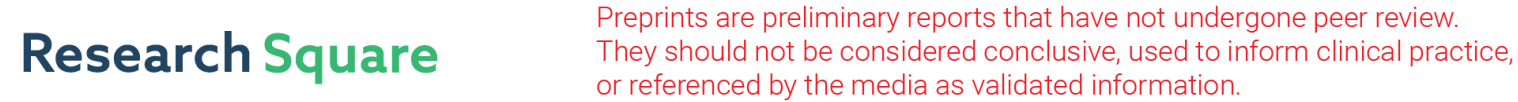 \\ All-optical control of pendular qubit states with nonresonant two-color laser pulses.
}

\author{
POSTECH \\ Minemoto Shinichirou \\ The University of Tokyo \\ Dong Eon Kim \\ POSTECH \\ Hirofumi Sakai \\ The University of Tokyo
}

Je Hoi Mun ( $\square$ mun1219@mpk.or.kr)

\section{Research Article}

Keywords: quantum qubit controls, quantum paths, nonresonant two-color laser pulses

Posted Date: November 15th, 2021

DOI: https://doi.org/10.21203/rs.3.rs-1028039/v1

License: (c) (1) This work is licensed under a Creative Commons Attribution 4.0 International License.

Read Full License

Version of Record: A version of this preprint was published at Communications Physics on September 14th, 2022. See the published version at https://doi.org/10.1038/s42005-022-01005-y. 


\title{
All-optical control of pendular qubit states with nonresonant two-color laser pulses
}

\author{
Je Hoi Mun ${ }^{1,2,3},{ }^{*}$ Shinichirou Minemoto ${ }^{1}$, Dong Eon Kim ${ }^{2,3}$, and Hirofumi Sakai ${ }^{1,4 \dagger}$ \\ ${ }^{1}$ Department of Physics, Graduate School of Science, \\ The University of Tokyo, 7-3-1 Hongo, Bunkyo-ku, Tokyo 113-0033, Japan \\ ${ }^{2}$ Department of Physics and Center for Attosecond Science and Technology, POSTECH, Pohang, 37673, South Korea \\ ${ }^{3}$ Max Planck POSTECH/KOREA Research Initiative, Pohang, 37673, South Korea and \\ ${ }^{4}$ Institute for Photon Science and Technology, Graduate School of Science, \\ The University of Tokyo, 7-3-1 Hongo, Bunkyo-ku, Tokyo 113-0033, Japan
}

(Dated: October 28, 2021)

\begin{abstract}
Practical methodologies for quantum qubit controls are established by two prerequisites, i.e., preparation of a well-defined initial quantum state and coherent control of that quantum state. Here we propose a new type of quantum control method, realized by irradiating nonresonant nanosecond two-color $(\omega$ and $2 \omega$ ) laser pulses to molecules in the pendular (field-dressed) ground state. The two-color field nonadiabatically splits the initial pendular ground state $|\tilde{0}, \tilde{0}\rangle$ to a superposition state of $|\tilde{0}, \tilde{0}\rangle$ and $|\tilde{1}, \tilde{0}\rangle$, whose relative probability amplitudes can be controlled by the peak intensity of one wavelength component $(\omega)$ while the peak intensity of the other component $(2 \omega)$ is fixed. The spliting of the quantum paths is evidenced by observing degrees of orientation of ground-stateselected OCS molecules by the velocity map imaging technique. This quantum control method is highly advantageous in that any type of polar molecules can be controlled regardless of the molecular parameters, such as rotational energy, permanent dipole moment, polarizability, hyperpolarizability, and hyperfine energy structures.
\end{abstract}

\section{INTRODUCTION}

Nowadays studies on controlling the molecular rota- ${ }^{54}$ tion can be devided into two big branches. One is re- ${ }^{55}$ lated to the molecular spectroscopy. Gas molecules can ${ }^{56}$ be fixed in the laboratory-fixed frame by irradiating a ${ }^{57}$ moderately strong laser field $[1,2]$. Such a molecular ${ }^{58}$ ensemble is referred to as being aligned. In the aligned ${ }^{59}$ molecular ensemble, the molecular angular distribution is ${ }^{60}$ symmetric with respect to the plus-minus inversion of the ${ }^{61}$ aligned axis. A molecular ensemble with the directional 62 asymmetry is called being oriented. There are a num- ${ }_{63}$ ber of studies on realization of strong molecular align- 64 ment and orientation by combined electrostatic and laser ${ }_{65}$ fields [3-5], a two-color laser field [6-10], and a THz laser 60 field $[11,12]$. The laser-induced molecular alignment ${ }_{67}$ and orientation techniques enabled photoelectron spectroscopy $[13,14]$ and high-harmornic spectroscopy [15$17]$ in molecule-fixed frames so that anisotropic properties including the molecular orbitals of sample molecules can be investigated.

The studies on molecular rotational dynamics are also 69 connected with another big branch of quantum science, ${ }^{70}$ so-called the quantum computer or quantum simulator [18], which has attracted widespread attention of many researchers in the relevant fields. Quantum computation has been pursued with various physical systems, ${ }_{73}$ including trapped cold ions [19], nuclear magnetic res- ${ }^{74}$ onance [20], quantum dots [21], and a superconducting ${ }_{75}^{74}$ circuit [22]. Also, the molecular rotational state of polar ${ }_{76}^{76}$ molecules was proposed as a quantum platform [23]. The

\footnotetext{
*mun1219@mpk.or.kr

† hsakai@phys.s.u-tokyo.ac.jp
}

d an external electrostatic or magnetic field. A resonant
microwave pulse can drive transitions between the pendular states $[24,25]$. The driving frequency, intensity, and the polarization need to be optimized, depending on the the molecular species and the external field conditions. The resonant microwave electric field is widely used for coherent excitation of the molecular qubit system [26].

In this work, theoretically and experimentally, we show a new type of qubit control methodology for general molecules. We use nonresonant nanosecond two-color laser pulses, which are capable of completely controlling transition amplitudes of two laser-field-dressed states regardless of the energy structure of molecules.

\section{THEORY OF ALL-OPTICAL QUBIT CONTROL METHOD BY NONRESONANT TWO-COLOR LASER PULSES}

We use atomic units unless otherwise stated. In the purely adiabatic process, an initial field-free rotational state of a molecule designated by $|J, M\rangle$ evolves into a field-dressed state, so-called a pendular state. Since the one-to-one correspondence between an initial fieldfree state and a field-dressed pendular state is physically ensured, a pendular state is conventionally expressed as $|\tilde{J}, \tilde{M}\rangle$ by using the corresponding initial quantum numbers $J$ and $M$.

According to the Landau-Zener formula [27], a nonadaibatic transition rate $\Gamma(t)$ between two pendular 


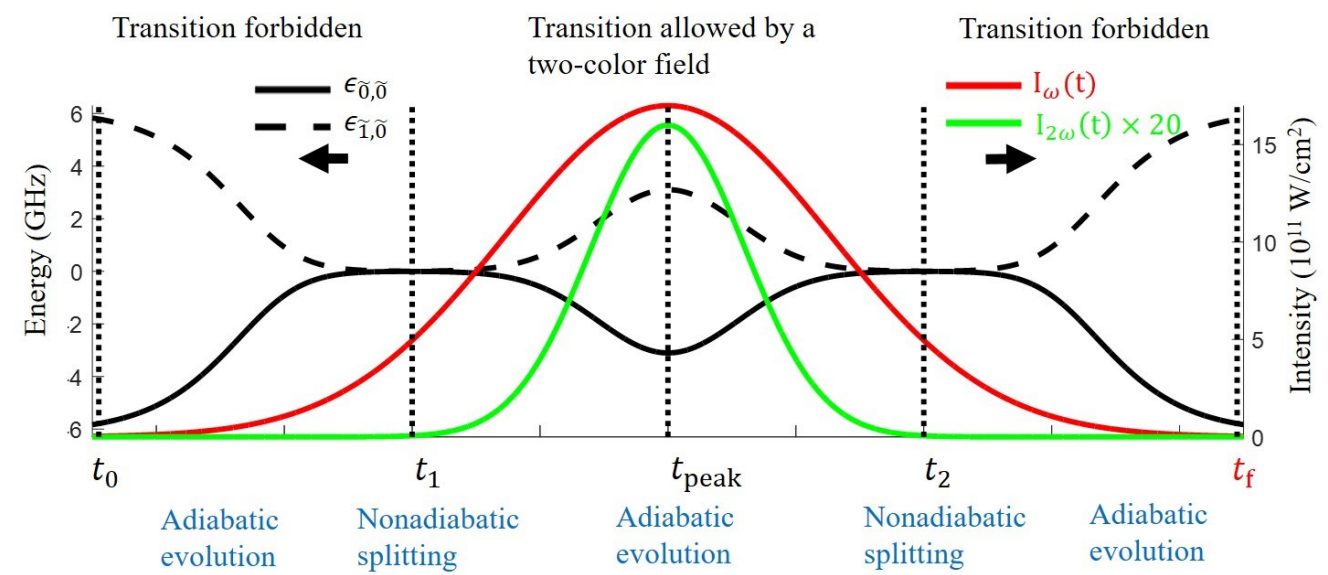

Initial state

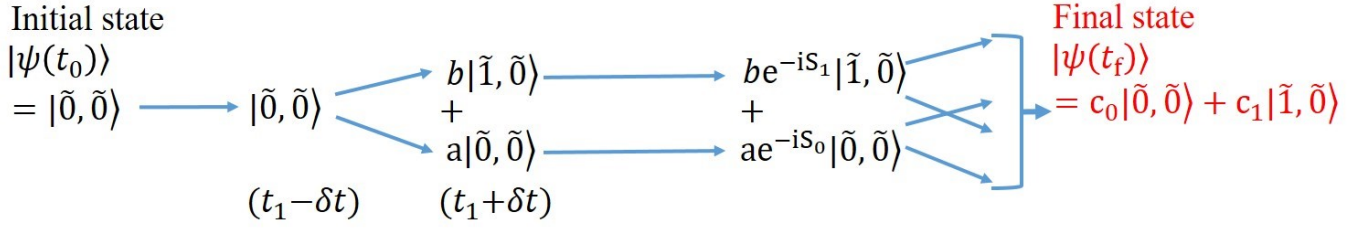

FIG. 1. Schematic illustration of qubit control by nonresonant two-color laser pulses. Temporal shapes of the $\omega$ and $2 \omega$ laser pulses and temporal variations of field-dressed eigenenergies $\left(\epsilon_{\tilde{n}, \tilde{0}}(n=0,1)\right)$ of the two pendular states are shown. Nonadiabatic splittings of the quantum state occur at the leading $\left(t_{1}\right)$ and the trailing $\left(t_{2}\right)$ edges of the $2 \omega$ laser pulse. Quantum path evolution of the initial pendular ground state $|\tilde{0}, \tilde{0}\rangle$ is schematically shown. The final state is given by equation (7). (See main text for the details.)

states is

$$
\Gamma(t)=\frac{d_{m n}^{2}}{\frac{\partial}{\partial t}\left(\epsilon_{m}-\epsilon_{n}\right)},
$$

where $\epsilon_{m(n)}$ is an energy of a field-dressed state la- ${ }_{106}$ belled by $m(n)$, and $d_{m n}$ is the transition matrix element ${ }_{107}$ between the two states. Formerly, with nonresonant ${ }_{108}$ nanosecond laser pulses with moderately strong inten-109 sities of $10^{10}-10^{12} \mathrm{~W} / \mathrm{cm}^{2}$, it was believed that molec- ${ }_{-110}$ ular rotational dynamics was adiabatic, because the ro- ${ }_{111}$ tational speed of molecules $\left(1 /\left(\epsilon_{m}-\epsilon_{n}\right)\right)$ is rapid enough $\mathrm{h}_{112}$ in comparison to the field intensity variation so that $\Gamma(t)_{113}$ is near zero. Therefore, the adiabaticity criterion can be $\mathrm{e}_{114}$ considered as $\Gamma(t) \sim 0$. Over the past years, however, it was revealed that nonadiabatic transitions between $\mathrm{a}_{115}$ pair of pendular states $|\tilde{0}, \tilde{0}\rangle$ and $|\tilde{1}, \tilde{0}\rangle$ can be significant $_{116}$ depending on the external field parameters [9, 10, 28-30].117 This intrinsically inevitable nonadiabatic transition be-118 tween pendular doublet states, though it is a technical ${ }_{119}$ issue to be overcome in the molecular orientation, can $_{120}$ be a powerful tool to control the qubit state given by $\mathrm{a}_{121}$ superposition of $|\tilde{0}, \tilde{0}\rangle$ and $|\tilde{1}, \tilde{0}\rangle$.

Figure 1 schematically shows our quantum control ${ }_{123}$ strategy. The second-harmonic pulse $(2 \omega)$ has a shorter ${ }_{124}$ pulse duration than the fundamental $(\omega)$ pulse, due to125 the second-order frequency conversion process. Therefore, in the leading edge of the nanosecond two-color laser pulse, virtually only the $\omega$ pulse interacts with molecules. By the $\omega$ field becoming strong enough, the molecules in both $|\tilde{0}, \tilde{0}\rangle$ and $|\tilde{1}, \tilde{0}\rangle$ states show strong alignment along the polarization direction of the $\omega$ field $[1,2]$. In this condition, the two states are not mixed because the nonresonant-laser-based alignment Hamiltonian has no transition matrix elements between the two states. In the presence of only the strong alignment potential, the two states are almost degenerate in energy at $t=t_{1}-\delta t$ as shown in Fig. 1. Up to this moment, the population of the initial pendular ground state $|\tilde{0}, \tilde{0}\rangle$ is preserved through the adiabatic process.

Combination of the $\omega$ and $2 \omega$ fields creates an orientation potential via hyperpolarizability interaction with the molecules $[6,7]$. As the $2 \omega$ intensity becomes significant, due to the orientation Hamiltonian, the transition matrix elements between the two states abruptly become nonzero. The degeneracy is clearly resolved as the $2 \omega$ intensity becomes stronger, and the two energy curves exhibit an avoided crossing at $t_{1}$ as shown in Fig. 1. Since the energy gap between the two states is near zero at the crossing point $t_{1}$, the two states can be mixed completely by the transition rate given by equation (1), such 

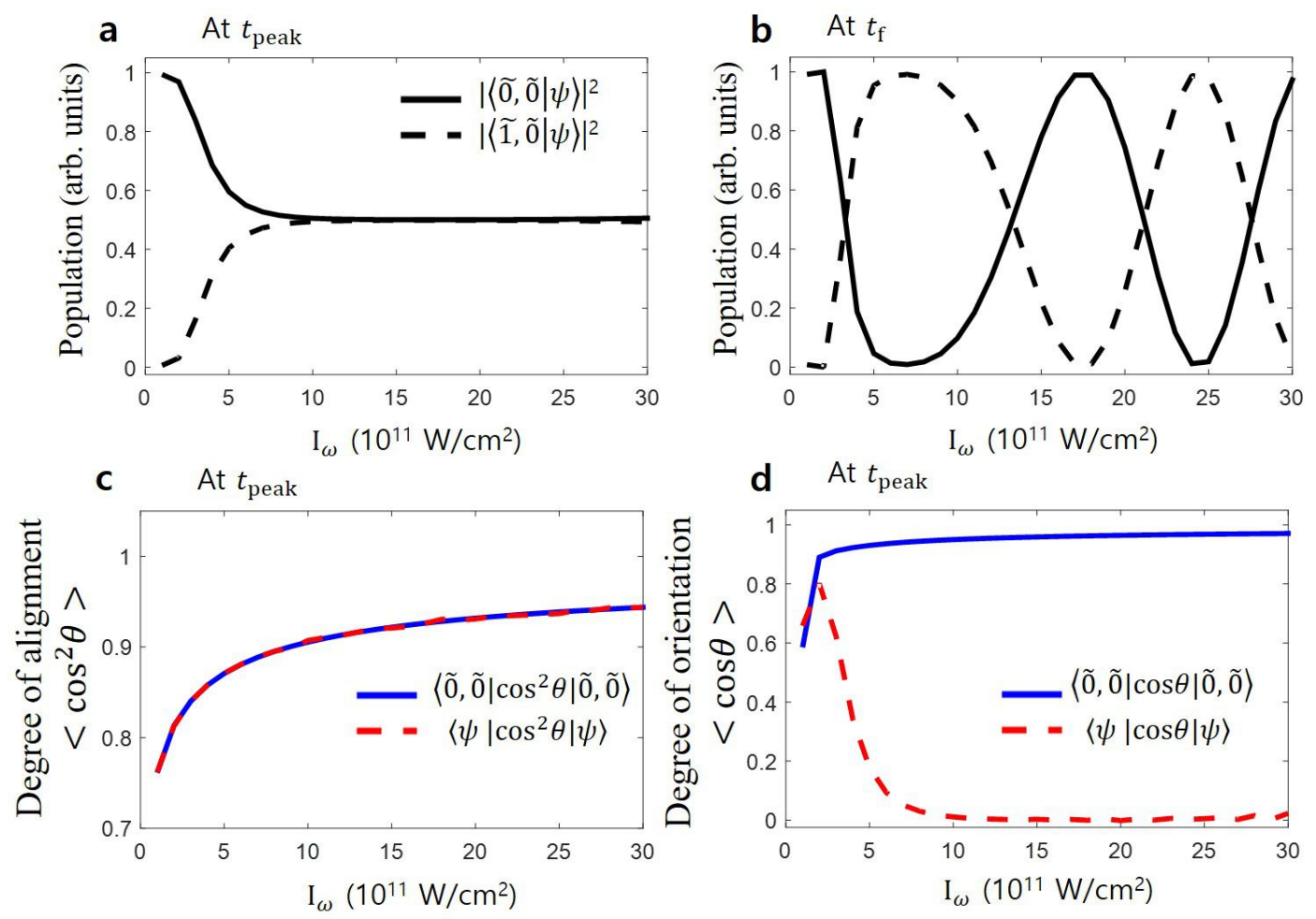

FIG. 2. Results of the relevant time-dependent Schrödinger equation. a, The pendular ground $|\tilde{0}, \tilde{0}\rangle$ (solid line) and excited $|\tilde{1}, \tilde{0}\rangle$ (dashed line) state populations at the peak $t_{\text {peak }}$ of the two-color pulse, and $\mathbf{b}$, those populations after the laser pulse has passed completely at $t_{\mathrm{f}}$. The degrees of alignment $\mathbf{c}$ and orientation $\mathbf{d}$ at $t_{\text {peak }}$ evaluated from the pendular ground state (blue-solid line) and the mixed pendular state $|\psi\rangle$ (red-dashed line) obtained by solving the TDSE. The results are shown as a function of the peak intensity $I_{\omega}$ of the $\omega$ pulse.

that the rotational state after $t=t_{1}+\delta t$ can be expressed as $a|\tilde{0}, \tilde{0}\rangle+b|\tilde{1}, \tilde{0}\rangle$ with $|a|^{2}=|b|^{2} \sim 1 / 2$. We note that, in the presence of the orientation potential, the two pendular states are oriented in the opposite directions. While each pendular state shows strong orientation, the degree of molecular orientation is virtually zero because the two pendular states are completely mixed. To increase the degree of orientation, it is desirable to temporally synchronize the alignment and orientation potentials by optimizing the delay between the $\omega$ and $2 \omega$ pulses $[9,10]$.

The two energy curves cross again in the trailing edge of the $2 \omega$ pulse at $t=t_{2}$. In between the crossing points of $t_{1}$ and $t_{2}$, the interaction can be considered adiabatic because the temporal variations of the energy curves are slow enough. Thus the adiabaticity criterion $\Gamma(t) \sim 0$ is satisfied.

At the second crossing point $t=t_{2}$, the quantum states experience another nonadiabatic splitting. After the splitting at $t=t_{2}$, the mixed quantum state adiabatically evolves until the laser pulses are completely turned off. Eventually, there are four quantum paths through the entire laser-molecule interaction process.

The final state $\left|\psi\left(t_{\mathrm{f}}\right)\right\rangle$ can be analytically expressed by using an initial state $\left|\psi\left(t_{0}\right)\right\rangle$ and the following unitary propagation operator.

$$
\hat{U}_{\mathrm{f}}=\hat{U}_{\mathrm{s}}^{\dagger} \hat{U}_{\mathrm{e}} \hat{U}_{\mathrm{s}}
$$

Here $\hat{U}_{\mathrm{s}}$ is a $2 \times 2$ unitary operator, nonadiabatically mixing an initial pendular ground state into a superposition state at time $t_{1}$. Another mixing process at $t_{2}$ can be given by the Hermitian conjugate of $\hat{U}_{\mathrm{s}}$ by assuming a temporally symmetric laser pulse. The unitary nature gives a $2 \times 2$ matrix in a generalized form, expressed as

$$
\hat{U}_{\mathrm{s}}=\left(\begin{array}{cc}
a & -b^{*} e^{i \chi} \\
b & a^{*} e^{i \chi}
\end{array}\right), \hat{U}_{\mathrm{s}}^{\dagger}=\left(\begin{array}{cc}
a^{*} & b^{*} \\
-b e^{-i \chi} & a e^{-i \chi}
\end{array}\right) .
$$

In between the nonadiabatic transition points of $t_{1}$ and $t_{2}$, the two adiabatic quantum paths accumulate phases, which can be considered by a diagonal matrix $\hat{U}_{\text {e }}$ given by

$$
\hat{U}_{\mathrm{e}}=\left(\begin{array}{cc}
e^{-i S_{0}} & 0 \\
0 & e^{-i S_{1}}
\end{array}\right), S_{n(n=0,1)} \equiv \int_{t_{1}}^{t_{2}} \epsilon_{|\tilde{n}, \tilde{0}\rangle} d t .
$$

Therefore, the total unitary operator is given by

$$
\hat{U}_{\mathrm{f}}=\left(\begin{array}{cc}
|b|^{2} e^{i Q}+|a|^{2} e^{-i Q} & a^{*} b^{*} e^{i \chi}\left[e^{i Q}-e^{-i Q}\right] \\
a b e^{-i \chi}\left[e^{i Q}-e^{-i Q}\right] & |a|^{2} e^{i Q}+|b|^{2} e^{-i Q}
\end{array}\right),
$$


where $Q \equiv\left(S_{0}-S_{1}\right) / 2$. In the right-hand side of equa-190 tion $(5), e^{-i P}\left(P \equiv\left(S_{0}+S_{1}\right) / 2\right)$ is omitted because it ${ }_{191}$ introduces a uniform phase shift to the operator. When ${ }_{192}$ $|\mathrm{a}|^{2}=|\mathrm{b}|^{2}=1 / 2$, equation (5) is

$$
\hat{U}_{\mathrm{f}}=\left(\begin{array}{cc}
\cos (Q) & 2 i a^{*} b^{*} e^{i \chi} \sin (Q) \\
2 i a b e^{-i \chi} \sin (Q) & \cos (Q)
\end{array}\right) .
$$

Eventually, the state $|\tilde{0}, \tilde{0}\rangle$ evolves into

$$
\cos (Q)|\tilde{0}, \tilde{0}\rangle+2 i a b e^{-i \chi} \sin (Q)|\tilde{1}, \tilde{0}\rangle
$$

and the state $|\tilde{1}, \tilde{0}\rangle$ evolves into

$$
2 i a^{*} b^{*} e^{i \chi} \sin (Q)|\tilde{0}, \tilde{0}\rangle+\cos (Q)|\tilde{1}, \tilde{0}\rangle
$$

by the irradiation of the nonresonant two-color laser $_{204}$ pulse. This result means that the final population in the $\mathrm{e}_{205}$ pendular ground state $|\tilde{0}, \tilde{0}\rangle$ and the first excited state $|\tilde{1}, \tilde{0}\rangle$ can be controlled by the quantum phase difference $Q=\int_{t_{1}}^{t_{2}}\left(\epsilon_{0}-\epsilon_{1}\right) d t$. The phase difference $Q\left(I_{\omega}\right)$ is $\operatorname{an}_{207}^{206}$ increasing function of the peak intensity of the $\omega$ pulse $_{208}$ because the energy gap of the two states for the time ${ }_{209}$ between $t_{1}$ and $t_{2}$ is an increasing function of the hy- ${ }_{210}$ perpolarizability interaction. Therefore, the final state ${ }_{211}$ $\left|\psi\left(t_{\mathrm{f}}\right)\right\rangle$ (shown in Fig. 1) can be controlled by the peak ${ }_{212}$ intensity $I_{\omega}$ of the $\omega$ pulse.

To validate this quantum control method, we have cal- ${ }_{214}$ culated the population dynamics by numerically solving ${ }_{215}$ the relevant TDSE $[9,10]$. An OCS molecule is used ${ }_{216}$ as a sample. Temporally synchronized $\omega$ and $2 \omega$ laser $_{217}$ pulses are used, whose pulse durations are set at $6 \mathrm{~ns}_{218}$ and $2 \mathrm{~ns}$ by FWHM, respectively. The polarizations of ${ }_{219}$ the two wavelengths are parallel. The ground state $|\tilde{0}, \tilde{0}\rangle_{220}$ is used as an initial condition. Numerical calculations ${ }_{221}$ are conducted by changing the peak intensity $I_{\omega}$ of the $\mathrm{e}_{222}$ $\omega$ pulse, while the peak intensity $I_{2 \omega}$ of the $2 \omega$ pulse is $_{223}$ fixed at $0.8 \times 10^{11} \mathrm{~W} / \mathrm{cm}^{2}$. Throughout the simulations, ${ }_{224}$ relative phase $\phi$ between the two wavelengths is set at $t_{225}$ zero to maximize the orientation potential, which is pro- $_{226}$ portional to $\cos \phi[6,7]$. Without loosing the concept ${ }_{227}$ of the underlying physical mechanism, the above simu- ${ }_{228}$ lation conditions are chosen to clearly demonstrate the ${ }_{229}$ nonadiabatic transitions at the leading and trailing edges ${ }_{230}$ of the $2 \omega$ pulse, so that the results are consistent with $_{231}$ the analytic solution.

Figure 2a shows the populations of the two states $_{233}$ $|\langle\tilde{0}, \tilde{0} \mid \psi\rangle|^{2}$ and $|\langle\tilde{1}, \tilde{0} \mid \psi\rangle|^{2}$ at the peak of the laser pulse, ${ }_{234}$ and those populations after the interaction are shown $\mathrm{in}_{235}$ Fig. 2b. At the peak intensity (Fig. 2a), more popula- ${ }_{236}$ tion transfer occurs by increasing $I_{\omega}$. The $|\tilde{1}, \tilde{0}\rangle$ state $_{237}$ population reaches near $50 \%$ at $I_{\omega}=1.0 \times 10^{12} \mathrm{~W} / \mathrm{cm}^{2}{ }_{, 238}$ which means that the two states are totally mixed in the 239 first nonadiabatic transition process at $t_{1}$. At the peak 240 intensity of the pulse, the degrees of alignment from the 241 ground state and the mixed state are consistent as shown 242 in Fig. 2c. However, while the ground state shows strong ${ }_{243}$ orientation, the mixed state shows significantly reduced ${ }_{244}$ orientation as shown in Fig. 2d because the two oppositely oriented states are mixed.

Looking at the populations after the laser pulse has completely passed (Fig. 2b), they show oscillating behaviors as expected from the analytic solution. When the peak intensity $I_{\omega}$ is above $1.0 \times 10^{12} \mathrm{~W} / \mathrm{cm}^{2}$ and the two states are completely mixed at the peak intensity of the laser pulse $\left(|a|^{2}=|b|^{2} \sim 1 / 2\right)$, the final populations oscillate between 0 and $100 \%$ as the peak $\omega$ intensity is further increased, which is consistent with the prediction from the analytic function (equation (7)). Creation of the four quantum paths and the interference between these paths cause the $I_{\omega}$-dependent modulation of the final state amplitudes.

\section{EXPERIMENTAL OBSERVATION OF THE NONADIABATIC TRANSITION}

Figure 3 shows a summary of our observations of molecular orientation by the velocity map imaging (VMI) technique. OCS molecules in the rotational ground state are used as a sample (see Supplementary information). At the peak intensity of the two-color pulse, the molecules are ionized by a femtosecond probe pulse as shown in Fig. 3a. The 2-dimensional velocity distributions of the $\mathrm{S}^{+}$fragment ions are observed, which reflect the angular distributions of the rotation-controlled neutral molecules. The typical $\mathrm{S}^{+}$ion images are shown in Figs. 3c-f. In the center of the images, there appear low energy fragment signals. These signals originate from dissociation of $\mathrm{OCS}^{+}$parent ions and are not a focus of the present study. In the concentric rings between 150 and 210 pixels, there appear signals upward and downward along the polarization direction. The up-down asymmetry along the polarization direction shows the evidence of orientation of OCS molecules. Orientation potential energies created by the two-color laser pulses are proportional to $\cos \phi$, with $\phi$ the relative phase between the two wavelengths (shown in Fig 3b). The $\cos \phi$ dependence of the orientation directions is seen in the comparisons between (c) and (d) or (e) and (f).

The $\mathrm{S}^{+}$ion images are measured by changing $\phi$. We evaluate the degrees of alignment $\left\langle\cos ^{2} \theta_{2 \mathrm{D}}\right\rangle(\mathrm{g})$ and orientation $\left\langle\cos \theta_{2 \mathrm{D}}\right\rangle(\mathrm{h})$ of the rotation-controlled neutral molecules from the ion distributions with $\theta_{2 \mathrm{D}}$ the angle between the polarization direction of the two-color control pulse and the detected ion direction. When $I_{\omega}$ is increased from $1.7 \times 10^{11} \mathrm{~W} / \mathrm{cm}^{2}$ to $5.0 \times 10^{11} \mathrm{~W} / \mathrm{cm}^{2}$, the degree of alignment is increased as shown in Fig. $3 \mathrm{~g}$, while the $\phi$-dependent modulation amplitude of the degree of orientation is reduced as shown in Fig. 3h. Note that the degree of alignment is independent of $\phi$ within data fluctuations and is increased when the $\omega$ intensity is increased, which ensures that the experimental conditions such as spatial overlap of the two-color beams are constant during the experiment. If the process is adiabatic, the degrees of alignment and orientation should 

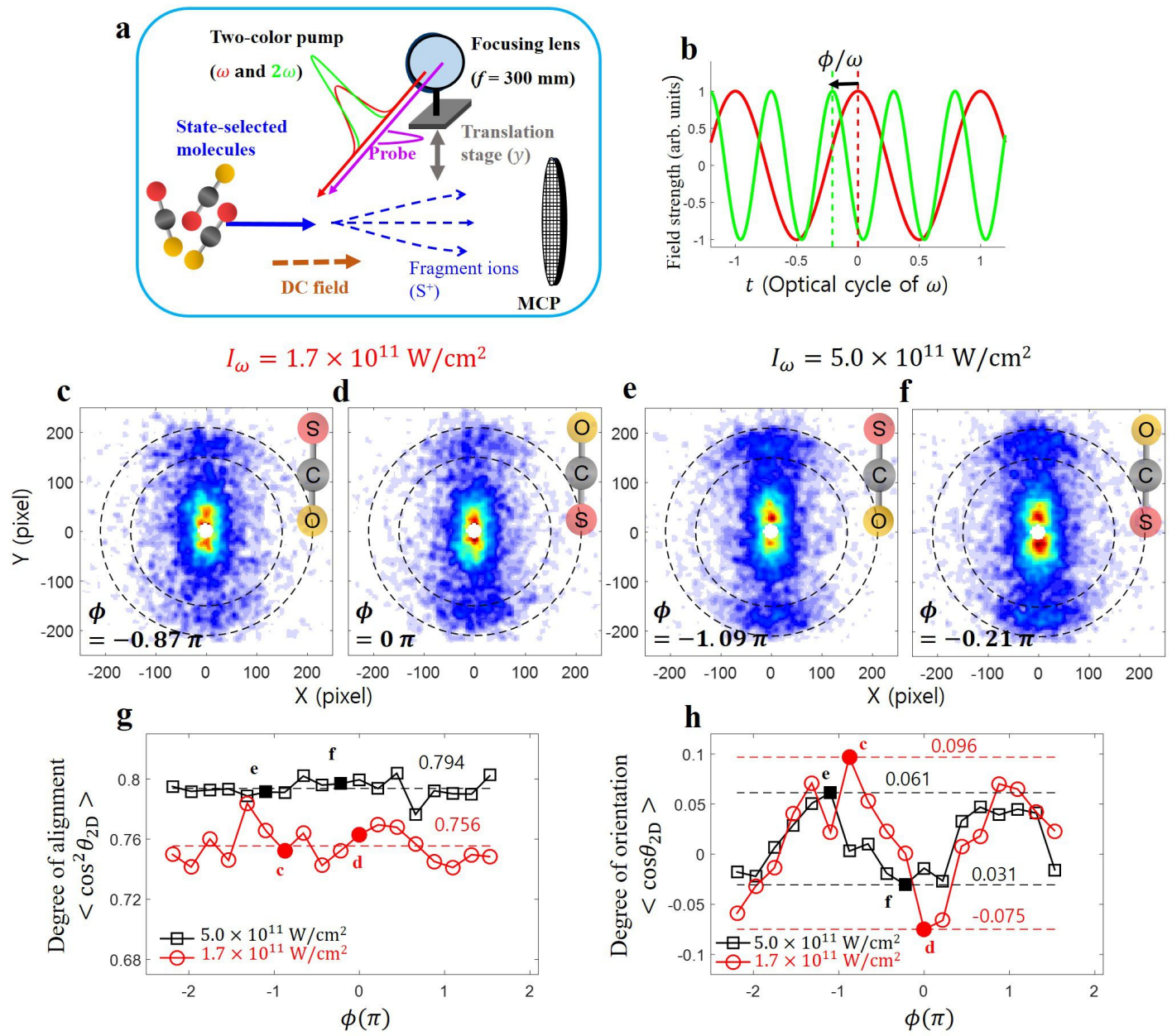

FIG. 3. Experimental observation of the nonadiabatic transition. a, Velocity-map imaging for observing the molecular orientation. The pump and probe pulses are irradiated to OCS molecules in the rotational ground state. b, Schematic representation of the relative phase $\phi$ between the two wavelengths. c,d,e,f, Typical images of the $\mathrm{S}^{+}$fragment ions observed in different conditions. $\mathbf{g}, \phi$-independent degrees of alignment $\left\langle\cos ^{2} \theta_{2 \mathrm{D}}\right\rangle$ and $\mathbf{h}, \phi$-dependent degrees of orientation $\left\langle\cos \theta_{2 \mathrm{D}}\right\rangle$ for two peak $\omega$ intensities of $5.0 \times 10^{11} \mathrm{~W} / \mathrm{cm}^{2}$ (black) and $1.7 \times 10^{11} \mathrm{~W} / \mathrm{cm}^{2}$ (red).

increase by increasing the peak intensity of the $\omega$ pulse.261 The reduced degree of orientation means that nonadia-262 batic transition between oppositely oriented two pendu-263 lar states actually takes place as discussed for Fig. 2d.264 Since the nonadiabatic process is observed in the first265 half of the two-color pulse, another Hermitian conjugate 266 nonadiabatic process should also take place in the second 267 half of the two-color pulse.

\section{METHOD}

As a pump pulse for the quantum control, the funda-273 mental $(\omega)$ and the second-harmonic $(2 \omega)$ pulses from $a^{274}$ injection seeded Nd:YAG laser (Spectra-Physics, LAB-275 130-10) are used. The duration of the $\omega$ pulse is $9 \mathrm{~ns}^{276}$ and that of the $2 \omega$ pulse is 6 ns by FWHM. The relative phase $\phi$ between the $\omega$ and the $2 \omega$ pulses is varied by changing the angle of a fused silica plate inserted in the beam path. The peak intensity of the $2 \omega$ pulse is fixed at $2.0 \times 10^{11} \mathrm{~W} / \mathrm{cm}^{2}$, while the $\omega$ intensity is changed by an attenuator consisting of a $\lambda / 2$ waveplate and a polarizer. As a probe pulse, output from a Ti:sapphire amplifier is used with the center wavelength of $800 \mathrm{~nm}$ and the pulse width of $35 \mathrm{fs}$. The polarizations of the pump pulses are set parallel to the detector plane and that of the probe pulse is perpendicular to the detector plane. The pump and the probe pulses are collinearly focused by a 300$\mathrm{mm}$ lens into the state-selected molecular beam. The $\mathrm{S}^{+}$fragment ions are detected by a microchannel plate (MCP) backed by a phosphor screen and the images on the screen are recorded by a charge-coupled device camera. In Figs. $3 g$ and h, both $\left\langle\cos ^{2} \theta_{2 \mathrm{D}}\right\rangle$ 's and $\left\langle\cos \theta_{2 \mathrm{D}}\right\rangle$ 's are evaluated based on the angular distributions observed for $\mathrm{S}^{+}$fragment ions as shown in Figs. 3c-f. 


\section{SUMMARY AND OUTLOOK}

In this study, using the ground-state-selected target ${ }^{297}$ OCS molecules, we demonstrated a new type of qubit ${ }^{298}$ control method realized by nonresonant two-color laser ${ }^{299}$ pulses, rather than a resonant microwave. This approach ${ }^{300}$ has a strong advantage in that it does not require ei- ${ }^{301}$ ther resonance frequency tuning or an external field, and ${ }^{302}$ the quantum control can be all-optically achieved. Uti- ${ }^{303}$ lizing nonresonant laser pulses brings us a capability of ${ }^{304}$ qubit control regardless of molecular energy structures. ${ }^{305}$ Thus, this all-optical nonresonant field approach has $a^{306}$ tremendous potential in coherent control of rotational ${ }^{307}$ qubit states. This study paves the way to control multiple qubits by various new methods utilizing nonresonant laser pulses.

\section{DATA AVAILABILITY}

The data that support the findings of this study are ${ }^{313}$ available from the corresponding authors upon reasonable request.
[1] B. Friedrich and D. Herschbach, Alignment and Trapping 346 of Molecules in Intense Laser Fields, Physical Review Let-347 ters 74, 4623 (1995).

[2] B. Friedrich and D. Herschbach, Polarization of 349 Molecules Induced by Intense Nonresonant Laser Fields, 350 The Journal of Physical Chemistry 99, 15686 (1995). 351

[3] H. Sakai, S. Minemoto, H. Nanjo, H. Tanji, and 352 T. Suzuki, Controlling the Orientation of Polar Molecules353 with Combined Electrostatic and Pulsed, Nonresonant354 Laser Fields, Physical Review Letters 90, 083001 (2003).355

[4] D. Takei, J. H. Mun, S. Minemoto, and H. Sakai, Laser-356 field-free three-dimensional molecular orientation, Phys-357 ical Review A 94, 013401 (2016).

[5] L. Holmegaard, J. H. Nielsen, I. Nevo, H. Stapelfeldt, 359 F. Filsinger, J. Küpper, and G. Meijer, Laser-Induced 360 Alignment and Orientation of Quantum-State-Selected 361 Large Molecules, Physical Review Letters 102, 023001362 (2009).

[6] T. Kanai and H. Sakai, Numerical simulations of molecu-364 lar orientation using strong, nonresonant, two-color laser 365 fields, The Journal of Chemical Physics 115, 5492 (2001).366

[7] K. Oda, M. Hita, S. Minemoto, and H. Sakai, All-367 Optical Molecular Orientation, Physical Review Letters368 104, 213901 (2010).

369

[8] K. Lin, I. Tutunnikov, J. Qiang, J. Ma, Q. Song, Q. Ji,370 W. Zhang, H. Li, F. Sun, X. Gong, H. Li, P. Lu, 371 H. Zeng, Y. Prior, I. S. Averbukh, and J. Wu, All-optical ${ }_{372}$ field-free three-dimensional orientation of asymmetric-373 top molecules, Nature Communications 9, 5134 (2018). 374

[9] J. H. Mun and H. Sakai, Improving molecular orientation 375 by optimizing relative delay and intensities of two-color
This study was supported by the National Research Foundation of Korea (NRF) grant funded by the Korea government (MSIT)[Grant No 2020R1C1C1012953]. It was also supported in part by the Max Planck POSTECH/KOREA Research Initiative Program through the National Research Foundation of Korea (NRF) funded by the Ministry of Science and ICT[Grant No 2016K1A4A4A01922028]. The experimental part of this study was supported by Grant-in-Aid for Specially Promoted Research No. 21000003 from MEXT and the Photon Frontier Network Program of MEXT.

\section{AUTHOR CONTRIBUTIONS}

J.H.M., S.M., D.E.K., and H.S. contributed to discussions and the preparation of the manuscript. J.H.M. performed the calculations. J.H.M. and S.M performed the experiments.

\section{COMPETING INTERESTS}

The authors declare no competing interests. laser pulses, Physical Review A 98, 013404 (2018).

[10] J. H. Mun, H. Sakai, and R. González-Férez, Orientation of linear molecules in two-color laser fields with perpendicularly crossed polarizations, Physical Review A 99, 053424 (2019).

[11] K. Kitano, N. Ishii, and J. Itatani, High degree of molecular orientation by a combination of $\mathrm{THz}$ and femtosecond laser pulses, Physical Review A 84, 053408 (2011).

[12] L. Xu, I. Tutunnikov, E. Gershnabel, Y. Prior, and I. S. Averbukh, Long-Lasting Molecular Orientation Induced by a Single Terahertz Pulse, Physical Review Letters 125, 013201 (2020).

[13] L. Holmegaard, J. L. Hansen, L. Kalhøj, S. Louise Kragh, H. Stapelfeldt, F. Filsinger, J. Küpper, G. Meijer, D. Dimitrovski, M. Abu-samha, C. P. J. Martiny, and L. Bojer Madsen, Photoelectron angular distributions from strong-field ionization of oriented molecules, Nature Physics 6, 428 (2010).

[14] M. Meckel, D. Comtois, D. Zeidler, A. Staudte, D. Pavičić, H. C. Bandulet, H. Pépin, J. C. Kieffer, R. Dörner, D. M. Villeneuve, and P. B. Corkum, LaserInduced Electron Tunneling and Diffraction, Science 320, 1478 (2008).

[15] J. Itatani, J. Levesque, D. Zeidler, H. Niikura, H. Pépin, J. C. Kieffer, P. B. Corkum, and D. M. Villeneuve, Tomographic imaging of molecular orbitals, Nature 432, 867 (2004).

[16] T. Kanai, S. Minemoto, and H. Sakai, Quantum interference during high-order harmonic generation from aligned molecules, Nature 435, 470 (2005). 
17] C. Vozzi, M. Negro, F. Calegari, G. Sansone, M. Nisoli,401 S. De Silvestri, and S. Stagira, Generalized molecular or-402 bital tomography, Nature Physics 7, 822 (2011).

[18] C. P. Koch, M. Lemeshko, and D. Sugny, Quantum con-404 trol of molecular rotation, Reviews of Modern Physics405 91, 035005 (2019).

19] H. Häffner, C. F. Roos, and R. Blatt, Quantum comput-407 ing with trapped ions, Physics Reports 469, 155 (2008).408

[20] J. A. Jones and M. Mosca, Implementation of a quan-409 tum algorithm on a nuclear magnetic resonance quan-410 tum computer, The Journal of Chemical Physics 109,411 1648 (1998).

[21] D. Loss and D. P. DiVincenzo, Quantum computation 413 with quantum dots, Physical Review A 57, 120 (1998). 414

[22] C. Song, S.-B. Zheng, P. Zhang, K. Xu, L. Zhang,415 Q. Guo, W. Liu, D. Xu, H. Deng, K. Huang, D. Zheng,416 $\mathrm{X}$. Zhu, and H. Wang, Continuous-variable geometric ${ }_{417}$ phase and its manipulation for quantum computation in a418 superconducting circuit, Nature Communications 8, $1061_{419}$ (2017).

420

[23] D. DeMille, Quantum Computation with Trapped Polar421 Molecules, Physical Review Letters 88, 067901 (2002). ${ }_{422}$

[24] S. A. Will, J. W. Park, Z. Z. Yan, H. Loh, and 423 M. W. Zwierlein, Coherent Microwave Control of Ultra-424 cold ${ }^{23} \mathrm{Na}^{40} \mathrm{~K}$ Molecules, Physical Review Letters 116,425
225306 (2016).

[25] J.-R. Li, W. G. Tobias, K. Matsuda, C. Miller, G. Valtolina, L. De Marco, R. R. W. Wang, L. Lassablière, G. Quéméner, J. L. Bohn, and J. Ye, Tuning of dipolar interactions and evaporative cooling in a three-dimensional molecular quantum gas, Nature Physics 17, 1144 (2021).

[26] L. D. Carr, D. DeMille, R. V. Krems, and J. Ye, Cold and ultracold molecules: science, technology and applications, New Journal of Physics 11, 055049 (2009).

[27] C. Wittig, The Landau-Zener Formula, The Journal of Physical Chemistry B 109, 8428 (2005).

[28] Y. Sugawara, A. Goban, S. Minemoto, and H. Sakai, Laser-field-free molecular orientation with combined electrostatic and rapidly-turned-off laser fields, Physical Review A 77, 031403(R) (2008).

[29] M. Muramatsu, M. Hita, S. Minemoto, and H. Sakai, Field-free molecular orientation by an intense nonresonant two-color laser field with a slow turn on and rapid turn off, Physical Review A 79, 011403(R) (2009).

[30] J. H. Nielsen, H. Stapelfeldt, J. Küpper, B. Friedrich, J. J. Omiste, and R. González-Férez, Making the Best of Mixed-Field Orientation of Polar Molecules: A Recipe for Achieving Adiabatic Dynamics in an Electrostatic Field Combined with Laser Pulses, Physical Review Letters 108, 193001 (2012). 


\section{Supplementary Files}

This is a list of supplementary files associated with this preprint. Click to download.

- SupplementaryV63.pdf 